INPLASY

PROTOCOL

To cite: Chae et al. The effectiveness and safety of Simiao Xiaobi decoction on rheumatoid arthritis: A study protocol for systematic review and meta-analysis. Inplasy protocol 202230026. doi: 10.37766/inplasy2022.3.0026

Received: 06 March 2022

Published: 06 March 2022

Corresponding author:

Won-Suk Sung

1984sws@hanmail.net

Author Affiliation:

Dongguk University Bundang Oriental Hospital.

Support: None.

Review Stage at time of this submission: The review has not yet started.

Conflicts of interest: None declared.

\section{The effectiveness and safety of Simiao Xiaobi decoction on rheumatoid arthritis : A study protocol for systematic review and meta-analysis}

Chae, SY1; Kim, EJ2; Park, SS3; Sung, WS4.

Review question / Objective: We are going to investigate the effectiveness and safety of Simiao Xiaobi decoction (SXD) for rheumatoid arthritis (RA).

Condition being studied: Several clinical trials including randomized controlled trials (RCTs) showed that SXD significantly relieved the clinical symptoms of RA comparing to the conventional treatment group.

Information sources: MEDLINE, Cochrane Library, China National Knowledge Infrastructure (CNKI), CiNii, J-STAGE, KoreaMed, Korean Medical Database, Korean Studies Information Service System (KISS), National Digital Science Library(NDSL), Korea Institute of Science and Technology Information (KISTI), and Oriental Medicine Advanced Searching Integrated System (OASIS) will be used to search for related studies published from initial time of each database to July 2022, There is no language restriction and the search will be performed according to the language provided by each database.

INPLASY registration number: This protocol was registered with the International Platform of Registered Systematic Review and Meta-Analysis Protocols (INPLASY) on 06 March 2022 and was last updated on 06 March 2022 (registration number INPLASY202230026).

\section{INTRODUCTION}

Review question / Objective: We are going to investigate the effectiveness and safety of Simiao Xiaobi decoction (SXD) for rheumatoid arthritis (RA).

Rationale: Conventional treatments for RA have limitations and other complementary and alternative medicines are gaining interest. Although several studies reported the effectiveness of SXD on RA, systematic review about it was not yet provided.

Condition being studied: Several clinical trials including randomized controlled trials (RCTs) showed that SXD significantly relieved the clinical symptoms of RA 
comparing to the conventional treatment group.

\section{METHODS}

Search strategy: We will search related RCT through multiple electronic databases, manual search, and contact to author.

Participant or population: Patients diagnosed as RA.

Intervention: Simiao Xiaobi decoction.

Comparator: Other conservative treatments for RA including placebo, medication, and physiotherapy. The usage of combination treatment with SXD in the experimental group should be consistent with control group.

Study designs to be included: RCTs that investigated the effects of SXD on RA with control group containing other conventional treatments.

Eligibility criteria: We will only include RCTs. Non-RCTs and uncontrolled clinical trials will be excluded. There are no restrictions on language or neither publications.

Information sources: MEDLINE, Cochrane Library, China National Knowledge Infrastructure (CNKI), CiNii, J-STAGE, KoreaMed, Korean Medical Database, Korean Studies Information Service System (KISS), National Digital Science Library(NDSL), Korea Institute of Science and Technology Information (KISTI), and Oriental Medicine Advanced Searching Integrated System (OASIS) will be used to search for related studies published from initial time of each database to July 2022, There is no language restriction and the search will be performed according to the language provided by each database.

Main outcome(s): Disease activity score including effective rate, swollen joint count, tender joint count, morning stiffness.
Additional outcome(s): Blood test about RA including ESR, CRP, and RF, and adverse events.

Quality assessment / Risk of bias analysis: Risk of bias will be assessed by two reviewers independently using the Chochrane "risk of bias" tool. The tool is composed of seven domains: sequence generation, allocation concealment, blinding of participants and investigators, blinding of outcome assessors, incomplete outcome data, selective outcome reporting, and other biases. The risk of bias will be rated as "low risk," "high risk," or "unclear risk" for each domain.

Strategy of data synthesis: We will use the Review Manager (RevMan) software for Windows to perform a meta-analysis (Version 5.3; Copenhagen; The Nordic Cochrane Center, The Cochrane Collaboration, 2014). We will combine the changes between baseline and intervention completion in the included studies. The mean difference (same outcome) or standardized mean difference (different outcome) and $95 \%$ confidence intervals will be calculated with random-effects model or a fixed-effect model.

Subgroup analysis: Potentially if data suitable based on the main intervention in control group.

Sensitivity analysis: We will perform a sensitivity analysis to test the robustness of study finding.

Country(ies) involved: Republic of Korea.

Keywords: rheumatoid arthritis, Simiao Xiaobi decoction, medication, systematic review, meta-analysis.

Contributions of each author:

Author 1 - Soo-Yeon Chae.

Author 2 - Eun-Jung Kim.

Author 3 - Seong-Sik Park.

Author 4 - Won-Suk Sung. 